\title{
Differential gene expression profile in spleen of mandarin fish Siniperca chuatsi infected with ISKNV, derived from suppression subtractive hybridization
}

\author{
Wei He $^{1, \dagger}$, Zhi-Xin Yin ${ }^{1, \dagger}$, You Li ${ }^{1}$, Wei-Li Huo ${ }^{1}$, Hao-Ji Guan ${ }^{1}$, Shao-Ping Weng ${ }^{1}$, \\ Siu-Ming Chan², Jian-Guo $\mathrm{He}^{1, *}$ \\ ${ }^{1}$ State Key Laboratory for Biocontrol, School of Life Sciences, Zhongshan University, 135 Xingang Road West, \\ Guangzhou 510275, PR China \\ ${ }^{2}$ Department of Zoology, The University of Hong Kong, Pokfulam Road, Hong Kong SAR, PR China
}

\begin{abstract}
To study the interaction between an invading virus and its host, we investigated differential gene expression in mandarin fish Siniperca chuatsi experimentally infected with infectious spleen and kidney necrosis virus (ISKNV). Subtractive cDNA libraries were constructed by suppression subtractive hybridization (SSH) from spleens of mock- and ISKNV-infected fish. Both forwardand reverse-subtracted libraries were generated. In the forward library, genes of the ubiquitinproteasome proteolytic pathway, defense-related genes, a cytoskeletal protein gene, an apoptosisrelated gene encoding inhibitor of apoptosis protein and JFC/EBPb cDNA for CAAT/Enhancer binding protein beta were up-regulated after infection. In the reverse library, genes that encoded CD59/neurotoxin/Ly-6-like protein, carboxypeptidase A2, and goose-type lysozyme were downregulated. Some of these genes were analyzed by reverse transcription-polymerase chain reaction to confirm their differential expression as a result of virus infection. The results of this study may contribute to our understanding of fish innate immune response to ISKNV.
\end{abstract}

KEY WORDS: Infectious spleen and kidney necrosis virus $\cdot$ ISKNV $\cdot$ Siniperca chuatsi $\cdot$ Suppression subtractive hybridization $\cdot \mathrm{SSH} \cdot$ Ubiquitin-proteasome proteolytic pathway $\cdot$ Apoptosis

\section{INTRODUCTION}

Iridoviruses are large ( 120 to $200 \mathrm{~nm})$ DNA viruses that can infect invertebrates and poikilothermic vertebrates, including insects, fishes, amphibians and reptiles (Williams 1996). The family Iridoviridae has been divided into 5 genera, including Iridovirus, Chloriridovirus, Ranavirus, Lymphocystisvirus and Megalocystivirus (http://phene.cpmc.columbia.edu/Ictv/ index.htm). In recent years, megalocystiviruses have attracted much attention because most of them can cause serious systemic diseases in a wide-range of economically important freshwater and marine fish species worldwide. Fish affected by the megalocystivirus include red sea bream Pagrus major (Inouye et al. 1992), sea bass Lateolabrax sp. (Nakajima \& Sorimachi 1995), brown-spot grouper Epinephelus tauvina (Chua et al. 1994), malabar grouper E. malabaricus (Danayadol et al. 1996), angelfish Pterophyllum scalare (Rodge et al. 1997), grouper Epinephelus sp. (Chou et al. 1998), tilapia Oreochromis niloticus (McGrogan et al. 1998), mandarin fish Siniperca chuatsi (He et al. 2000), African lampeye Aplocheilichthys normani (Sudthongkong et al. 2002a), dwarf gourami Colisa lalia (Sudthongkong et al. 2002b), red drum Sciaenop socellata (Weng et al. 2002), rock bream Oplegnathus fasciatus (Jung \& Oh 2000), large yellow croaker Larimichthys croce (Chen et al. 2003) and turbot 
Scophthalmus maximus (Shi et al. 2004). In a recent investigation of host range performed in the South China Sea, 13 cultured and 39 wild marine fish species were confirmed hosts of the megalocystiviruses (Wang et al. 2006).

Infectious spleen and kidney neurosis virus (ISKNV) is the type species of the genus Megalocystivirus. It can infect the mandarin fish Siniperca chuatsi Basilewsky, which will develop serious systemic disease (He et al. 2002). The virus infection is capable of causing severe epizootics resulting in mass mortalities. The mandarin fish is the main farmed fish species in China with an annual output reaching 140000 tons and valued at 1 billion US dollars in 2003. In the last decade, the spread of ISKNV has resulted in significant economic losses for many fish farms (He et al. 1998, 2000). Therefore, preventing and controlling the spread of megalocystivirus has become a priority to the fish industry.

An infectious disease is the manifestation of a dynamic series of events that occur between the host and pathogen, which are defined by the interaction of pathogen-expressed virulence factors and the surveillance and defense systems of the host. Presently, much remains to be elucidated about host-megalocystivirus interactions at the molecular level. In this study, a sensitive PCR-based subtraction approach, suppression subtractive hybridization (SSH) (Diatchenko et al. 1996) was adopted to isolate genes up- or down-regulated in ISKNV-infected mandarin fish and identify their expression profile. cDNAs from the forward and reverse subtracted libraries were sequenced and analyzed. We identified some genes in the spleen of the mandarin fish that may be involved in the defense against ISKNV. These results will help to understand the infectious mechanism of the virus and the defense mechanism of fish, and perhaps lead to the prevention of virus outbreaks in the fish industry.

\section{MATERIALS AND METHODS}

Fish and ISKNV infection. Mandarin fish were obtained from fish farms in Nanhai, Guangdong Province, China, and kept in an aquarium with fresh water at $28^{\circ} \mathrm{C}$ for more than $1 \mathrm{wk}$ before the experiments. Six fish (weight $200.2 \pm 38.1 \mathrm{~g}$, body length 24.1 $\pm 1.2 \mathrm{~cm}_{\text {; }}$ mean $\pm \mathrm{SD}$ ) were randomly selected from these fish and kept in separate $40 \mathrm{l}$ aquaria. Tank water was passed through a sand-filter with a carbon layer and aerated before use.

To ensure the fish were not infected by ISKNV initially, peripheral blood was collected from the caudal artery and genomic DNA was extracted using the Blood Genomic DNA Purification System (Sino-American Biotechnology, China) according to the manu- facturer's instructions. A pair of primers (F1: 5'CATCAGCAGACCGAGCGTAA-3', R1: 5'-CCACCTGCTGTTTGCGAGT-3') were designed according to the VEGF gene of ISKNV and used to amplify the virus gene fragment $(596 \mathrm{bp})$. The final PCR mixture $(50 \mu \mathrm{l}$

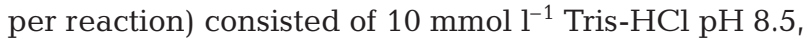
$1.8 \mathrm{mmol} \mathrm{l}^{-1} \mathrm{MgCl}_{2}, 50 \mathrm{mmol} \mathrm{l}^{-1} \mathrm{KCl}$, dNTP mixture (0.2 $\mathrm{mmol} \mathrm{l}^{-1}$ each), $10 \mathrm{pmol}$ of primers each and an aliquot of the genomic DNA. The PCR conditions were 26 cycles of $94^{\circ} \mathrm{C}$ for $30 \mathrm{~s}, 55^{\circ} \mathrm{C}$ for $30 \mathrm{~s}$ and $72^{\circ} \mathrm{C}$ for $1 \mathrm{~min}$. At the end of the last cycle, the tubes were further incubated at $72^{\circ} \mathrm{C}$ for 10 min for the completion of DNA synthesis. The whole blood genomic DNA from fish infected with ISKNV (confirmed by histopathology) was used as a positive control. The PCR products were analyzed on $1 \%$ agarose gel. All 6 fish were confirmed ISKNV negative.

Virus preparations were prepared as previously described (He et al. 2002). Briefly, spleens $(20 \mathrm{~g})$ of moribund mandarin fish experimentally infected with ISKNV were homogenized with phosphate-buffered saline (PBS), $\mathrm{pH} 7.2$, and centrifuged at $1500 \times g$ for $30 \mathrm{~min}$ at $4^{\circ} \mathrm{C}$. The supernatant was supplemented with $1000 \mathrm{IU} \mathrm{ml}^{-1}$ penicillin and $1000 \mathrm{mg} \mathrm{ml}^{-1}$ streptomycin and filtered through a $0.22 \mathrm{~mm}$ membrane. The experimental fish were infected with ISKNV by intramuscular injection of $1 \mathrm{ml}$ of filtrate. A $50 \mu \mathrm{l}$ aliquot of the filtrate was inoculated on tryptone soya agar for bacteriological examination. No bacteria were detected after 7 d at $28^{\circ} \mathrm{C}$. We injected 3 fish with ISKNV preparations. The other 3 fish were injected with the same volume of PBS ( $\mathrm{pH} 7.2$ ) as controls.

RNA isolation and cDNA synthesis. On the 4th day after injections, all the fish were sacrificed and the spleens removed aseptically for dissection. The spleens from each group of fish were pooled and stored in RNAlater (Ambion, USA) at $-20^{\circ} \mathrm{C}$ until further use. The anterior kidneys were dissected, genome DNA was extracted and ISKNV infection determined by PCR. Total RNA was extracted with Trizol reagent (Invitrogen, USA) according to the manufacturer's instructions. The integrity of the total RNA was routinely checked by electrophoresis of $20 \mu \mathrm{g}$ total RNA through $1 \%$ agarose, $6.5 \%$ formaldehyde gels in $1 \times$ MOPS (3-N-morpholino-2-hydroxypropane sulfonic acid) buffer. Complement DNA was synthesized from total RNA using the Super SMART PCR cDNA Synthesis Kit (Clontech, USA). Briefly, $1 \mu \mathrm{g}$ of total RNA was mixed with $7 \mu \mathrm{l}$ of $12 \mu \mathrm{M}$ 3' SMART CDS Primer IIA and $7 \mu \mathrm{l}$ of $12 \mu \mathrm{mol}^{-1}$ SMART IIA oligonucleotide. The mixture was incubated at $72^{\circ} \mathrm{C}$ for $2 \mathrm{~min}$. Then, $20 \mu \mathrm{l}$ of $5 \times$ First-strand buffer, $2 \mu \mathrm{l}$ of $100 \mathrm{mmol} \mathrm{l}^{-1}$ DTT, $10 \mu \mathrm{l}$ of $50 \times$ dNTP $\left(10 \mathrm{mmol} \mathrm{l}^{-1}\right.$ each), $2.5 \mu \mathrm{l}$ of RNase inhibitor $\left(40 \mathrm{U} \mathrm{\mu l}^{-1}\right)$ and $5 \mu \mathrm{l}$ of PowerScript Reverse transcriptase (Clontech, USA) were added to the mixture (total 
$100 \mu \mathrm{l})$ and incubated at $42^{\circ} \mathrm{C}$ for $90 \mathrm{~min}$. Then, $2.5 \mu \mathrm{l}$ of the first strand cDNA product was combined with $10 \mu \mathrm{l}$ of $10 \times$ Advantage PCR buffer, $2 \mu \mathrm{l}$ of $50 \times$ dNTP, $2 \mu \mathrm{l}$ of $5 \times$ PCR Primer IIA, $2 \mu \mathrm{l}$ of $50 \times$ Advantage polymerize mix and $81.5 \mu$ of deionized water. The mixture was then amplified by PCR for 18 cycles $\left(94^{\circ} \mathrm{C}\right.$ for $30 \mathrm{~s}$ and $68^{\circ} \mathrm{C}$ for $6 \mathrm{~min}$ each). The PCR product was used for the following SSH experiment.

Suppression subtractive hybridization. SSH was performed with the PCR-select cDNA Subtraction Kit (Clontech, USA). Briefly, Super SMART cDNA from the PBS-injected (Driver) and ISKNV-infected (Tester) fish was digested with restriction endonuclease RsaI to generate short, blunt-ended double-strand cDNAs. After phenol extraction and ethanol precipitation, RsaI-digested tester cDNA was separately ligated with Adaptor 1 and Adaptor 2R at the 5'-end of each strand of the tester cDNA. After denaturation at $95^{\circ} \mathrm{C}$ for $90 \mathrm{~s}$, the adaptor 1 ligated and adaptor 2R ligated tester cDNA were then separately hybridized with an excess of driver cDNA at $68^{\circ} \mathrm{C}$ for $8 \mathrm{~h}$. The 2 hybridization samples were mixed together without denaturation and hybridized at $68^{\circ} \mathrm{C}$ overnight with an excess of fresh denatured driver cDNA. The resulted mixture was diluted to $200 \mu \mathrm{l}$ then amplified by 2 rounds of PCR to enrich the desired cDNAs containing both adaptors by exponential amplification of these products. The primary PCR was performed with flanking primers against adaptor 1 and adaptor $2 \mathrm{R}$, and the amplified products were used as template in the secondary PCR with nested primers. Finally, the efficiency was evaluated by PCR with primers for the $\alpha$-actin gene (forward: 5'-AGTCCCCTCCCCCGAAACAC-3'; reverse: 5'-GGGCCCACCAGAGAATAAACCTT-3') performed on tester (unsaturated) and subtracted cDNA for 20,25 , and 30 cycles. In the reverse subtraction experiment, PBS-injected fish cDNA was used as tester, while ISKNV-injected fish cDNA was used as driver.

Cloning, sequencing and BLAST homology search. The PCR products from the subtraction procedures were cloned into pDrive Cloning Vector (Qiagen, Germany) to create the forward and reverse subtracted cDNA libraries. The libraries were transformed into TG1 competent cells, followed by plating onto agar plates containing ampicillin, X-gal (5-bromo-4-chloro-3-indolyl- $\beta$-D-galactopyranoside), and IPTG (isopropyl- $\beta$-D-thiogalactopyranoside). A total of 275 and 111 randomly selected white clones were sequenced, from the forward and reverse libraries respectively. These sequences were edited by using programs to remove vector sequences and ambiguous regions, and then assembled into groups of sequences (clusters) using the SEQMAN subprogram of DNASTAR software (Madison, WI, USA) so as to assemble the longest possible consensus sequences. The consensus sequences of each cluster were used as query sequences to search the non-redundant protein database at the National Center for Biotechnology Information (NCBI) with BLASTx (Benson et al. 2000). For those sequences with no 'hits' found, BLASTn was used to search for similar nucleotide sequences.

Criteria for identification of clones. In those cases for which BLASTx analysis returned E-values < E-5 (indicative of a significant homology), it was common to find significant similarities ('hit') to several sequences in the database. The identifications we assigned were made as follows: if the similarity was markedly higher for one 'hit', that was chosen as the identification of our sequence. In some cases in which E-values were similar for a number of 'hits', we examined the list for molecules from teleost fish and these identifications were assigned. For some sequences that did not meet the criteria of E $<\mathrm{E}-5$, if they showed $>90 \%$ amino acid identity with known genes or sequences, we assigned the identities to the similar genes.

RT-PCR. RT-PCR was performed to compare the differential expression level of 28 genes in spleen PBS and ISKNV-injected fish. The 18s rRNA gene was used as control. The primers for target genes and 18s rRNA gene are listed in Table 1. Total RNA was prepared from the spleens of fish. $2 \mu \mathrm{g}$ of total RNA was mixed with $2 \mu \mathrm{l}$ of $12 \mu \mathrm{mol} \mathrm{ml}^{-1} 3^{\prime}$ SMART CDS Primer IIA and $1 \mu \mathrm{l}$ of random primer ( 9 mers, $1 \mathrm{mg} \mathrm{ml}^{-1}$ ). The mixture was incubated at $72^{\circ} \mathrm{C}$ for $2 \mathrm{~min}$. Then, $5 \mu \mathrm{l}$ of $5 \times$ Moloney murine leukemia virus reverse transcriptase (MMLV) buffer, $0.5 \mu \mathrm{l}$ of $100 \mathrm{mmol}^{-1} \mathrm{DTT}, 2 \mu \mathrm{l}$ of dNTP (10 mmol $\mathrm{l}^{-1}$ each), $0.5 \mu \mathrm{l}$ of RNase inhibitor $\left(40 \mathrm{U} \mathrm{\mu l}^{-1}\right)$ and 1 Unit of MMLV (Promega, USA) were added to the mixture (total $20 \mu \mathrm{l}$ ) and incubated at $42^{\circ} \mathrm{C}$ for 90 min for the cDNA synthesis. Equal amounts of cDNAs from the PBS and ISKNV-injected samples were used as template in PCR. The PCR included 28 to 29 cycles of $94^{\circ} \mathrm{C}$ for $30 \mathrm{~s}, 52^{\circ} \mathrm{C}$ for $30 \mathrm{~s}$ and extension at $72^{\circ} \mathrm{C}$ for $40 \mathrm{~s}$. The products were analyzed on $1 \%$ agarose gel.

\section{RESULTS}

\section{Suppression subtractive hybridization}

All the fish injected with ISKNV were confirmed by PCR to be infected by this virus (data not shown). To identify the mRNAs that are up- and down-regulated in ISKNV-infected fish, SSH was performed using spleens of PBS and ISKNV-injected fish. After 2 rounds of hybridization and 2 rounds of PCR to amplify the differentially expressed cDNA, a forward subtraction library and a reverse subtraction library were constructed. We evaluated the subtraction efficiency using the constitutively expressed $\alpha$-actin gene. After 25 
Table 1. Primers used in semi-quantitative RT-PCR used to confirm differences in ISKNV expression levels in mandarin fish Siniperca chuatsi

\begin{tabular}{|c|c|c|}
\hline Primer & Sequence $\left(5^{\prime}-3^{\prime}\right)$ & $\begin{array}{l}\text { Product } \\
\text { size (bp) }\end{array}$ \\
\hline 18S-F & ATGGTACTTTAGGCGCCTAC & 280 \\
\hline $18 \mathrm{~S}-\mathrm{R}$ & TATACGCTATTGGAGCTGG & \\
\hline IAP-F & CATCGTTTTCATCCCATGTGGACACC & 115 \\
\hline IAP-R & TGAGAGGAAGGTTCGGACCGTGCC & \\
\hline Ubiquitin-activating enzyme E1-F & TTTGAGAAGGATGACGACGGTAACT & 242 \\
\hline Ubiquitin-activating enzyme E1-R & AGGTTGATATAAGCTGTGCGGTAAGA & \\
\hline Interferon-inducible protein Gig 2-F & CACCAGGAAGGCTGCTCAGTCCA & 241 \\
\hline Interferon-inducible protein Gig 2-R & CACCGGGCCGTATCTGGAGTCAT & \\
\hline ISKNV-induced protein 1-F & CTCGGGTCCGGGGATTGTTCCCT & 197 \\
\hline ISKNV-induced protein 1-R & TTTCTCCAGACGCTCACTGTCTT & \\
\hline CD59-F & ACACCTGTGCCAAAATTACCTACCC & 143 \\
\hline CD59-R & TTATTTGTTGCAGCCGTCCCAGTTG & \\
\hline Polyubiquitin-F & ACCCGACGAGACTGTGAGCGACTT & 131 \\
\hline Polyubiquitin-R & CTTCGACGTTGTAGTCCGCAAGT & \\
\hline Ribosomal protein L10F & AAGACCTGCGGAAAGGA & 122 \\
\hline Ribosomal protein L10R & GCACCACGCATTCCAGT & \\
\hline Ribosomal protein L10a-F & GCGGGGCTTTTCATC & 141 \\
\hline Ribosomal protein L10a-R & CTCCACCGACTCCACAA & \\
\hline Ribosomal protein L17-F & GCACATCCAGGTCAACAA & 128 \\
\hline Ribosomal protein L17-R & GGACGATCTGCTCCTTCT & \\
\hline Ribosomal protein L17-2-F & ATCAAGGGGCGTCTCAA & 122 \\
\hline Ribosomal protein L17-2-R & CGCTGCCGTATCACCA & \\
\hline Ribosomal protein L19-F & AGGCTGAGGCTCGTCG & 152 \\
\hline Ribosomal protein L19-R & CACTTGTCAGGCAGGATTG & \\
\hline Ribosomal protein L21-F & TGCTGTCGGCATCATCG & 189 \\
\hline Ribosomal protein L21-R & GCAGGCTGGCGTTTCA & \\
\hline Ribosomal protein L22-F & AAGCAGGTGGTCAAGAAGC & 166 \\
\hline Ribosomal protein L22-R & CACCGCCAAGGTTTCC & \\
\hline Ribosomal protein L30-F & ACGGACTAAACCGCAAAA & 141 \\
\hline Ribosomal protein L30-R & CCCCTGACGGATCATCTT & \\
\hline Ribosomal protein S3a-F & GCCTGTCAGTCCATCTACC & 180 \\
\hline Ribosomal protein S3a-R & CTCATAACCATCAGCCCTC & \\
\hline Ribosomal protein S5-F & GCAACAACGGCAAGAAG & 146 \\
\hline Ribosomal protein S5-R & ACCAATACGGGTGGAGTC & \\
\hline Ribosomal protein S7-F & GACGCCATCTTGGAGGA & 175 \\
\hline Ribosomal protein S7-R & CGTCTTTGCCTGTGAGC & \\
\hline Ribosomal protein S14-F & AAACGTGTTCGGAGTCTGT & 120 \\
\hline Ribosomal protein S14-R & TCCGCCTTCACCTTCAT & \\
\hline Ribosomal protein S19-F & GGAAGTCGCCAGAGGAA & 172 \\
\hline Ribosomal protein S19-R & GGCCAGCAATTCTATCCAG & \\
\hline Ribosomal protein S29-F & GGCTTTCCGACGCTC & 157 \\
\hline Ribosomal protein S29-R & GCACTGGCGGCACAT & \\
\hline Carboxypeptidase A2-F & CCAGTGGCGGCAGCATTGA & 131 \\
\hline Carboxypeptidase A2-R & GGTCTCGGAGGCAGTGGGGA & \\
\hline Mitochondrial ribosomal protein L11-F & AGTGAGGGCAGTGTATGAGA & 137 \\
\hline Mitochondrial ribosomal protein L11 -R & GATCGTTGACAACCTTGATG & \\
\hline Ubiquinol-cytochrome $c$ reductase core-F & TGGCAGACACATCCTGAACTACGG & 177 \\
\hline Ubiquinol-cytochrome $c$ reductase core- $R$ & CGCATTCTGTTGTAGTCGGGTAGC & \\
\hline Interleukin-8 variant-F & CAACTCCTTTCTACCATCGCCACC & 135 \\
\hline Interleukin-8 variant- $\mathrm{R}$ & GCTGGGCTTTAGAGATGCAGACCA & \\
\hline Transmembrane protein $7-\mathrm{F}$ & TGCTACGGAGATGAGGACGACGA & 125 \\
\hline Transmembrane protein $7-\mathrm{R}$ & TCATCCTGACAGCAAATGCCCA & \\
\hline Pleiotropic regulator 1-F & GAGGACGACACTGCCACGGAA & 418 \\
\hline Pleiotropic regulator 1-R & GGAGAGTGGCTGATGTTGGTGGAT & \\
\hline AY909489-unknown protein-F & CAGGAGTCCAGAGGAAACAGCCA & 157 \\
\hline AY909489-unknown protein-R & CGTCTAGTCCTTCTGCTGCTCCAA & \\
\hline AY909493- unknown protein-F & ATTGCCCTGCTGCCCAACATA & 140 \\
\hline AY909493-unknown protein-R & GGGAAGTTGCCTTGGCAGTATTCT & \\
\hline
\end{tabular}

PCR cycles, the $\alpha$-actin transcript was hardly detectable in the subtracted library compared to that in the unsubtracted sample, suggesting that the SSH procedure successfully suppressed cDNA common to the PBS and ISKNV-injected fish (Fig. 1).

The initial 275 expression sequence tags (ESTs) from the forward SSH library were grouped into 112 consensus sequences, consisting of 49 clusters that contained more than 2 ESTs per cluster and 63 singletons. Thirty-one of the consensus sequences showed significance with known genes (Table 2). They include genes of the ubiquitin-proteasome proteolytic (UPP) pathway (such as those encoding proteasome activator subunit 2, ubiquitin-activating enzyme E1 and polyubiquitin), defence-related genes (such as those encoded ferritin subunits, interferon-inducible protein Gig2, viperin, Mx protein, CC chemokine, hemolytic toxin, interleukin-8 and major histocompatibility class I receptor), an apoptosis-related gene encoding inhibitor of apoptosis protein, a cytoskeletal protein gene (talin) and $\mathrm{JFC} / \mathrm{EBPb} \mathrm{cDNA}$ for CAAT/Enhancer binding protein beta. For one particular sequence, the BLASTx analysis returned no 'hit'. Further BLASTn analysis returned positive hits of JFC/EBPb mRNA for CAAT/Enhancer binding protein beta, with the E-value 2E-72. The similarity was located at the 3 ' untranslated region of the target gene. Fragments similar to the polyubiquitin and the interferon-inducible protein Gig 2 were highly abundant in this library, with 11 clones for each.

The 111 ESTs from the reverse library were grouped into 55 consensus sequences, consisting of 17 clusters and 38 singletons. Thirty-two of these consensus sequences showed similarities to other genes (Table 3). They include 17 ribosomal protein genes, 2 cytoskeletal protein genes (tropomyosin4-1 and actinin-associated LIM protein), and those encoded for CD59/ neurotoxin/Ly-6-like protein, carboxypeptidase A2, zinc finger protein, testican 3, nascent-polypeptide-associated complex alpha polypeptide, and 


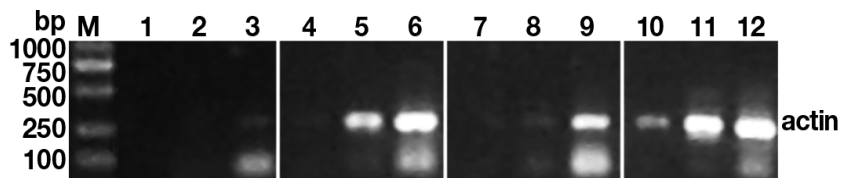

Fig. 1. Evaluation of the subtraction efficiency of subtracted cDNAs. PCRs with $\alpha$-actin-gene-specific primers were performed on cDNAs subtracted for forward library (lanes 1-3), unsubtracted for forward library (lanes 4-6), subtracted for reverse library (lanes 7-9) and unsubtracted for reverse library (lanes 10-12). PCRs were performed for 20 cycles (lanes 1, 4, 7 \& 10), 25 cycles (lanes 2, 5, 8 \& 11), and 30 cycles (lanes 3, 6, 9 $\& 12)$, respectively. The PCR products were electrophoresed on $1 \%$ agarose gel. M: DL-2000 DNA ladder (Shenerg Biocolor, Shanghai, China)

goose-type lysozyme. Fragments similar to the CD59/ neurotoxin/Ly-6-like protein were the most abundant in this library, with 7 clones.

The identified sequences were deposited to GenBank. Among these sequences, 6 are first found in fish, including phosphoribosyl pyrophosphate synthetase 1, testican 3, guanine nucleotide binding protein beta subunit 4, solute carrier family 29 (nucleoside transporters) member 4, DnaJ homolog subfamily C member 9 and talin.

From these consensus sequences, the putative complete open reading frames of 2 cDNAs were isolated: CD59/Ly-6/neurotoxin-like protein (GenBank Accession No. AY909432) and 60S ribosomal protein L30 (GenBank Accession No. AY909412).

\section{Confirmation of SSH by RT-PCR}

To confirm the result of $\mathrm{SSH}$, different clones were selected for RT-PCR. After normalization with constitutively expressed gene (i.e. 18s rRNA), the expression level of all the 12 genes from the forward library showed an apparent increase in expression in ISKNVinfected fish spleen (Fig. 2). These genes included polyubiquitin, ubiquinol-cytochrome $c$ reductase core I protein, interferon-inducible protein Gig2, ubiquitinactivating enzyme E1, transmembrane protein 7 , inhibitor of apoptosis protein, pleiotropic regulator 1 and ISKNV-induced protein 1. On the other hand, the expression level of all 16 genes from the reverse library was apparently decreased (Fig. 3). These included CD59/neurotoxin/Ly6, carboxypeptidase A2, and some ribosomal proteins.

\section{DISCUSSION}

Although the immune system has evolved mechanisms to eradicate viral-infected cells, many viruses can persist inside the cells to cause latent or chronic

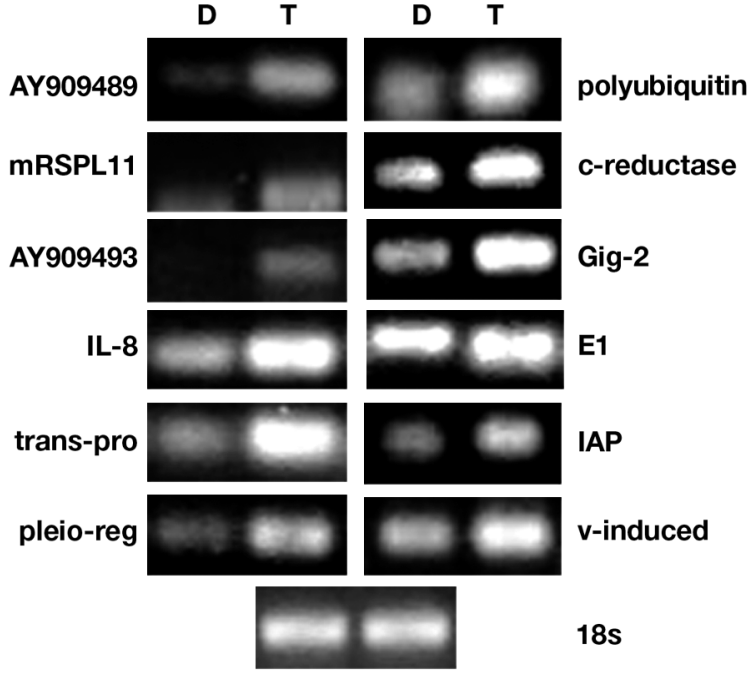

Fig. 2. Comparison of putative up-regulated genes between PBS- and ISKNV-injected fish using RT-PCR. D: PBS-injected fish; T: ISKNV-injected fish; mRSPL11: mitochondrial ribosomal protein L11; c-reductase: ubiquinol-cytochrome c reductase core I protein; Gig-2: interferon-inducible protein Gig2; IL-8: interleukin-8; E1: ubiquitin-activating enzyme E1; trans-pro: similar to transmembrane protein 7 ; IAP: inhibitor of apoptosis protein; pleio-reg: pleiotropic regulator 1 ; v-induced: ISKNV-induced protein 1; AY909493: unknown protein (GenBank Access. No. AY909493); AY909489: unknown protein (GenBank Access. No. AY909489)

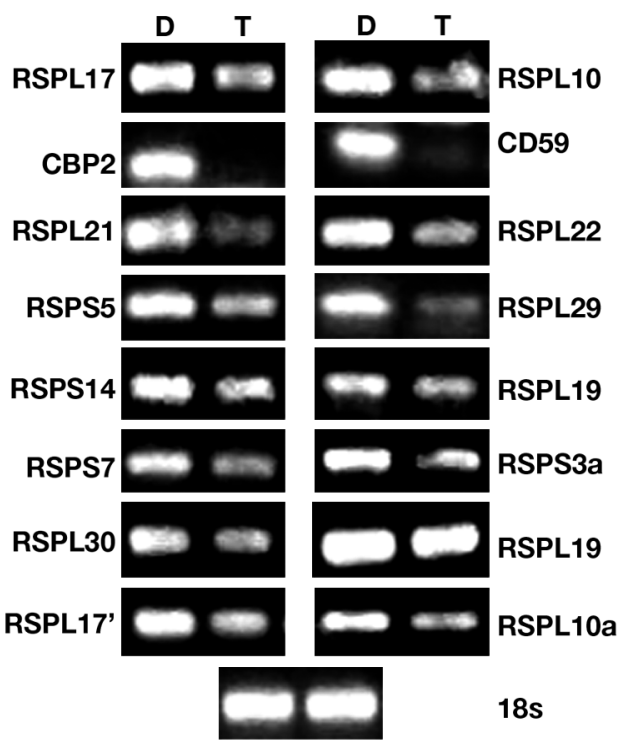

Fig. 3. Comparison of putative down-regulated genes between PBS- and ISKNV-injected fish using RT-PCR. D: PBSinjected fish; T: ISKNV-injected fish; 18s: 18s rRNA; RSPL10: 60s ribosomal protein L10; CBP-2: carboxypeptidase A2; CD59: CD59/Neurotoxin/Ly-6-like gene; RSPL21: 60s ribosomal protein L21; RSPL22: 60s ribosomal protein L21; RSPS5: 40s ribosomal protein S5; RSPS29: 40s ribosomal protein S29; RSPS14: 40s ribosomal protein S14; RSPS19: 40s ribosomal protein S19; RSPS7: 40s ribosomal protein S7; RSPS3a: 40s ribosomal protein S3a; RSPL30: 60S ribosomal protein L30; RSPL19: 60S ribosomal protein L19. RSPL17': 60s ribosomal protein L17 (AY909404); RSPL10a: 60s ribosomal protein L10a; RSPL17: 60s ribosomal protein L17 (AY909403) 
Table 2. Putative up-regulated genes identified in a cDNA library prepared by SSH using spleens of mandarin fish Siniperca chuatsi $4 \mathrm{~d}$ after ISKNV-injection. $\mathrm{x}$ : number of identical amino acids between query and subject sequences; $\mathrm{y}$ : number of amino acids for alignment

\begin{tabular}{|c|c|c|c|c|}
\hline Access. no. & $\begin{array}{l}\text { Closest database homologue } \\
\text { (Access. no.) }\end{array}$ & Species & $\begin{array}{l}\text { Longest fragment } \\
\text { (bp) (n) }\end{array}$ & $\begin{array}{l}\text { E Value } x / y \\
\text { (\% identity) }\end{array}$ \\
\hline AY909523 & VHSV-induced protein (AAM18479) & Oncorhynchus mykiss ${ }^{\mathrm{a}}$ & $421(3)$ & 2.00E-06 31/71 (43\%) \\
\hline AY909461 & $\begin{array}{l}\text { Interferon-inducible protein Gig2 } \\
\text { (AAP49829) }\end{array}$ & Carassius auratus $^{\mathrm{a}}$ & $637(11)$ & $3.00 \mathrm{E}-2458 / 85(68 \%)$ \\
\hline AY395718 & Viperin (AY395718) & Siniperca chuatsi ${ }^{\mathrm{a}}$ & $475(3)$ & 3.00E-57 131/131 (100\%) \\
\hline AY392097 & Mx protein (AAQ91382) & Siniperca chuatsi ${ }^{\mathrm{a}}$ & $429(4)$ & $2.00 \mathrm{E}-77142 / 142(100 \%)$ \\
\hline AF327363 & $\begin{array}{l}\text { Immunoglobulin heavy chain } \\
\text { (AF327363) }\end{array}$ & Siniperca chuatsi ${ }^{\mathrm{a}}$ & $303(1)$ & 4.00E-50 98/98 (100\%) \\
\hline AY909517 & CC chemokine (CAC45063) & Oncorhynchus mykiss ${ }^{\mathrm{a}}$ & $278(5)$ & 1.00E-14 35/62 (56\%) \\
\hline AY909475 & Interleukin-8 variant 5 (AAN41457) & Ictalurus punctatus ${ }^{\mathrm{a}}$ & $406(1)$ & $6.00 \mathrm{E}-0622 / 78(28 \%)$ \\
\hline AY909521 & Hemolytic toxin (BAC45007) & Phyllodiscus semoni & $244(1)$ & $9.00 \mathrm{E}-0430 / 84(35 \%)$ \\
\hline DQ058109 & Ubiquitin-like protein (AAO14689) & Oncorhynchus mykiss ${ }^{\mathrm{a}}$ & $229(11)$ & 7.00E-13 37/63 (58\%) \\
\hline DQ058110 & Cytochrome b (BAC99035) & Siniperca scherzeri ${ }^{\mathrm{a}}$ & $219(1)$ & 2.00E-20 51/72 (70\%) \\
\hline AY909514 & $\begin{array}{l}\text { Phosphoribosyl pyrophosphate } \\
\text { synthetase I (XP_420181) }\end{array}$ & Gallus gallus & $436(1)$ & $4.00 \mathrm{E}-73$ 140/145 (96\%) \\
\hline AY909449 & $\begin{array}{l}\text { Ubiquinol-cytochrome c reductase } \\
\text { core I protein (AAL74192) }\end{array}$ & Oncorhynchus mykiss ${ }^{\mathrm{a}}$ & $386(4)$ & 2.00E-47 88/95 (92\%) \\
\hline AY909450 & $\begin{array}{l}\text { Proteasome activator subunit } 2 \\
\text { (AAN40737) }\end{array}$ & Paralichthys olivaceus ${ }^{\mathrm{a}}$ & $395(1)$ & 2.00E-31 63/75 (84\%) \\
\hline AY909479 & $\begin{array}{l}\text { Similar to transmembrane protein } 7 \\
\text { (XP_236656) }\end{array}$ & Rattus norvegicus & $287(1)$ & 1.00E-06 28/87 (32\%) \\
\hline AY909447 & $\begin{array}{l}\text { Ubiquitin-activating enzyme E1 } \\
\text { (NP_003325) }\end{array}$ & Homo sapiens & $264(4)$ & $6.00 \mathrm{E}-24$ 51/86 (59\%) \\
\hline AY909520 & FK506 binding protein 5 (CAD87815) & Danio rerio ${ }^{\mathrm{a}}$ & $593(2)$ & $2.00 \mathrm{E}-26$ 60/96 (62\%) \\
\hline AY909477 & $\begin{array}{l}\text { Guanine nucleotide binding protein } \\
\text { beta subunit } 4 \text { (AAG18442) }\end{array}$ & Homo sapiens & $257(1)$ & 4.00E-39 74/76 (97\%) \\
\hline AY909485 & $\begin{array}{l}\text { Similar to human opioid growth } \\
\text { factor receptor (CAD44463) }\end{array}$ & Danio rerio ${ }^{\mathrm{a}}$ & $232(1)$ & 2.00E-21 46/68 (67\%) \\
\hline AY909445 & $\begin{array}{l}\text { Major histocompatibility class I } \\
\text { receptor (AAL11413) }\end{array}$ & Stizostedion vitreum ${ }^{\mathrm{a}}$ & $477(1)$ & 2.00E-67 119/158 (75\%) \\
\hline DQ058111 & $\begin{array}{l}\text { JFC/EBPb mRNA for CAAT/ } \\
\text { Enhancer binding protein } \\
\text { beta (AB049813) }\end{array}$ & Paralichthys olivaceus ${ }^{\mathrm{a}}$ & $258(1)$ & $\begin{array}{l}2.00 \mathrm{E}-72177 \mathrm{nt} / 189 \mathrm{nt} \\
(93 \%)(\mathrm{BLASTn})\end{array}$ \\
\hline AY909484 & Pleiotropic regulator 1 (AAQ91262) & Danio rerio & $615(1)$ & 6.00E-10 28/30 (93\%) \\
\hline AY909530 & $\begin{array}{l}\text { Solute carrier family } 29 \text { (nucleoside } \\
\text { transporters) member } 4 \text { (AAH47592) }\end{array}$ & Homo sapiens & $184(2)$ & 8.00E-07 24/34 (70\%) \\
\hline AY909531 & $\begin{array}{l}\text { DnaJ homolog, subfamily C, } \\
\text { member } 9 \text { (NP_598842) }\end{array}$ & Mus musculus & $382(2)$ & 9.00E-37 73/115 (63\%) \\
\hline AY909493 & $\begin{array}{l}\text { Hypothetical protein FLJ11354 } \\
\text { (NP_077024) }\end{array}$ & Homo sapiens & $295(1)$ & 3.00E-05 19/46 (41\%) \\
\hline AY909512 & $\begin{array}{l}\text { Inhibitor of apoptosis protein } \\
\text { (AAP04483) }\end{array}$ & Danio rerio ${ }^{\mathrm{a}}$ & $431(1)$ & 2.00E-23 48/49 (97\%) \\
\hline AY909429 & $\begin{array}{l}\text { Similar to mitochondrial riboso } \\
\text { mal protein L11 (XP_215146) }\end{array}$ & Rattus norvegicus & $168(1)$ & 7.00E-12 34/55 (61\%) \\
\hline AY909491 & Talin (AAF27330) & Homo sapiens & $271(1)$ & 2.00E-26 57/59 (96\%) \\
\hline AY909489 & $\begin{array}{l}\text { Unnamed protein product } \\
\text { (CAF91137) }\end{array}$ & Tetraodon nigroviridis ${ }^{\mathrm{a}}$ & $232(1)$ & $1.00 \mathrm{E}-1642 / 53(79 \%)$ \\
\hline AY909433 & Reverse transcriptase (AAC24982) & Synthetic construct & $348(1)$ & 6.00E-06 20/58 (34\%) \\
\hline AY909435 & $\begin{array}{l}\text { Reverse transcriptase-like protein } \\
\text { (AAD19348) }\end{array}$ & Takifugu rubripes ${ }^{\mathrm{a}}$ & $343(5)$ & $4.00 \mathrm{E}-1436 / 85(42 \%)$ \\
\hline
\end{tabular}

infection to the host. The identification of host genes induced by viruses is one of the critical steps in the elucidation of the host defense pathway. To study the interaction between ISKNV and the host mandarin fish, forward and reverse subtractive libraries were constructed from the fish spleen. After ISKNV infection, 31 putative up-regulated genes were identified in the spleen. On the other hand, 32 genes were downregulated. The major genes identified in this study are described below. 
Table 3. Putative down-regulated genes identified in a cDNA library prepared by SSH using spleens of mandarin fish Siniperca chuatsi $4 \mathrm{~d}$ after ISKNV-injections. $\mathrm{x}$ : number of identical amino acids between query and subject sequences; $\mathrm{Y}$ : number of amino acids for alignment

\begin{tabular}{|c|c|c|c|c|}
\hline Access. no. & $\begin{array}{l}\text { Closest database homologue } \\
\text { (Access. no.) }\end{array}$ & Species & $\begin{array}{l}\text { Longest fragment } \\
\text { (bp) (n) }\end{array}$ & $\begin{array}{l}\text { E Value } x / y \\
\text { (\% identity) }\end{array}$ \\
\hline AY738131 & Goose-type lysozyme (AY738131) & Siniperca chuatsi ${ }^{\mathrm{a}}$ & $133(1)$ & $6.00 \mathrm{E}-18$ 42/44 (95\%) \\
\hline AAQ14862 & AAQ14862) & Siniperca chuatsi ${ }^{\mathrm{a}}$ & $212(1)$ & $2.00 \mathrm{E}-1028 / 35(80 \%)$ \\
\hline AY909463 & Ferritin heavy subunit (AAB34575) & Salmo salar ${ }^{\mathrm{a}}$ & $387(4)$ & 3.00E-66 116/128 (90\%) \\
\hline AY909468 & Hemoglobin beta-A chain (Q9PVM2) & Seriola quinqueradiata ${ }^{\mathrm{a}}$ & $535(4)$ & 2.00E-69 127/146 (86\%) \\
\hline AY909469 & Hemoglobin beta-A chain (Q9PVM2) & Seriola quinqueradiata ${ }^{\mathrm{a}}$ & $513(5)$ & $2.00 \mathrm{E}-70127 / 146(86 \%)$ \\
\hline AY909470 & Hemoglobin alpha chain (P02018) & Carassius auratus $^{\mathrm{a}}$ & $196(2)$ & 8.00E-08 24/29 (82\%) \\
\hline AY909458 & Testican 3 (XP_224788) & Rattus norvegicus & 309 (1) & $3.00 \mathrm{E}-1838 / 52(73 \%)$ \\
\hline AY909432 & $\begin{array}{l}\text { Neurotoxin/CD59/Ly-6-like protein } \\
\text { (AAR20998) }\end{array}$ & Ctenopharyngodon idella ${ }^{\mathrm{a}}$ & $423(7)$ & $1.00 \mathrm{E}-0934 / 92(36 \%)$ \\
\hline AY909496 & Carboxypeptidase A2 (BAC53787) & Paralichthys olivaceus ${ }^{\mathrm{a}}$ & $535(1)$ & 3.00E-23 49/61 (80\%) \\
\hline AY909538 & $\begin{array}{l}\text { Nascent-polypeptide-associated } \\
\text { complex alpha polypeptide (AAP20156) }\end{array}$ & Pagrus major ${ }^{\mathrm{a}}$ & $102(1)$ & $0.7117 / 17(100 \%)$ \\
\hline AY909506 & Zinc finger (NP_938184) & Danio rerio ${ }^{\mathrm{a}}$ & $177(1)$ & $4.00 \mathrm{E}-14$ 35/48 (72\%) \\
\hline AY909403 & 60S ribosomal protein L17 (AAF61071) & Paralichthys olivaceus ${ }^{\mathrm{a}}$ & $441(1)$ & 6.00E-66 123/137 (89\%) \\
\hline AY909404 & 60S ribosomal protein L17 (AAC96111) & Dicentrarchus labrax $^{a}$ & $177(2)$ & $2.00 \mathrm{E}-2558 / 58(100 \%)$ \\
\hline AY909401 & 60S ribosomal protein L10a (AAK95136) & Ictalurus punctatus ${ }^{\mathrm{a}}$ & $299(2)$ & $4.00 \mathrm{E}-3774 / 84(88 \%)$ \\
\hline AY909424 & 40S ribosomal protein S17 (NP_001012) & Homo sapiens & $498(3)$ & $4.00 \mathrm{E}-48$ 95/97 (97\%) \\
\hline AY909417 & $40 \mathrm{~S}$ ribosomal protein s3a (O73813) & Oryzias latipes ${ }^{\mathrm{a}}$ & $283(1)$ & $4.00 \mathrm{E}-2861 / 78(78 \%)$ \\
\hline AY909422 & 40S ribosomal protein S14 (AAK95196) & Ictalurus punctatus ${ }^{\mathrm{a}}$ & $262(1)$ & $2.00 \mathrm{E}-33$ 69/71 (97\%) \\
\hline AY909426 & 40S ribosomal protein S23 (NP_001016) & Homo sapiens & $315(2)$ & $2.00 \mathrm{E}-45$ 91/92 (98\%) \\
\hline AY909425 & 40S ribosomal protein S19 (AAP20214) & Pagrus major ${ }^{\mathrm{a}}$ & $271(1)$ & $9.00 \mathrm{E}-38$ 78/81 (96\%) \\
\hline AY909416 & 40S ribosomal protein S8 (NP_001003) & Homo sapiens & $483(1)$ & $2.00 \mathrm{E}-46$ 86/94 (91\%) \\
\hline AY909399 & 60S ribosomal protein L10 (AĀK95135) & Ictalurus punctatus ${ }^{\mathrm{a}}$ & $194(1)$ & $9.00 \mathrm{E}-3163 / 64(98 \%)$ \\
\hline AY909408 & 60S ribosomal protein L22 (BAB21247) & Gallus gallus & $281(1)$ & 1.00E-28 61/65 (93\%) \\
\hline AY909406 & 60S ribosomal protein L19 (AAH62844) & Danio rerio ${ }^{\mathrm{a}}$ & $282(1)$ & 6.00E-06 23/23 (100\%) \\
\hline AY909418 & 40S ribosomal protein S5 (AAP20199) & Pagrus major ${ }^{\mathrm{a}}$ & $256(2)$ & 2.00E-42 84/84 (100\%) \\
\hline AY909412 & 60S ribosomal protein L30 (P58372) & Ictalurus punctatus ${ }^{\mathrm{a}}$ & 469 (1) & 4.00E-49 95/97 (97\%) \\
\hline AY909428 & 40S ribosomal protein S29 (AAK95214) & Ictalurus punctatus ${ }^{\mathrm{a}}$ & $173(2)$ & 2.00E-22 45/45 (100\%) \\
\hline AY909419 & 40S ribosomal protein s7 (P50894) & Takifugu rubripes ${ }^{\mathrm{a}}$ & $287(1)$ & 2.00E-34 72/74 (97\%) \\
\hline AY909407 & 60S ribosomal protein L21 (AAK95147) & Ictalurus punctatus ${ }^{\mathrm{a}}$ & $356(3)$ & 6.00E-55 101/114 (88\%) \\
\hline AY909515 & Tropomyosin4-1(BAC57571) & Takifugu rubripes ${ }^{\mathrm{a}}$ & $230(1)$ & 5.00E-32 70/76 (92\%) \\
\hline AY909526 & $\begin{array}{l}\text { Actinin-associated LIM protein } \\
\text { (CAC33787) }\end{array}$ & Salmo salar & $607(2)$ & 7.00E-83 145/185 (78\%) \\
\hline AY909483 & Thoc1 with death domain (AAH54938) & Danio rerio $^{\mathrm{a}}$ & $195(1)$ & 7.00E-17 42/52 (80\%) \\
\hline AY909532 & Unnamed protein (CAF99686) & Tetraodon nigroviridis ${ }^{\mathrm{a}}$ & $182(1)$ & 1.00E-09 34/51 (66\%) \\
\hline
\end{tabular}

\section{Cytokine genes}

Among the earliest immune mediators produced upon virus infection, cytokines orchestrate the induction and maintenance of innate and adaptive antiviral responses (Tortorella et al. 2000). Cytokines are powerful antiviral mediators, allowing the clearance of virus infection. After ISKNV infection, the expressions of Mx protein, interferon-inducible protein Gig-2, and viperin (interferon-inducible and antiviral protein) increased. Mx protein is an interferon-induced GTP-binding protein with documented antiviral activity (Leong et al. 1998). The increased expression of these genes suggested that the interferon pathway was stimulated after ISKNV infection. While several interferon cDNAs have been cloned from such fishes as goldfish (GenBank \#AY452069), Japanese puffer fish Takifugu rubripes (GenBank \#AJ583023), spotted green puffer fish Tetraodon nigrovirides (Lutfalla et al. 2003), Atlantic salmon (Robertsen et al. 2003), channel catfish (Long et al. 2004) and zebrafish (Altmann et al. 2003), the mandarin fish interferon cDNA has not been cloned. Two inflammatory cytokine genes, CC chemokine and interleukin-8 (CXC chemokine), were found in the forward SSH library. They are the major mediators of the inflammatory response, which indicates that inflammatory response might be essential in the defense of the fish. CD59/Neurotoxin/Ly-6-like protein gene was down-regulated after ISKNV infection. Mammalian CD59 is a complement regulatory protein, which can inhibit complement activation and membrane attack complex (MAC) formation on autologous cells (Fisicaro et al. 2000). Some virus infections decrease the expression levels of host CD59 (Aries et al. 1997, Kawano et al. 
1997, Rautemaa et al. 2002). Deficiency or down-regulation of CD59 results in unrestricted autologous MAC formation and, thereby, in higher sensitivity to complement attack. The down-regulation of CD59 in the ISKNV-infected host cells may make these cells more sensitive to complement attack. This may work as an anti-virus mechanism of the host. Further work should be done to confirm this. In the meantime, anaemia was demonstrated to be an important clinical symptom of ISKNVinfected mandarin fish (Weng et al. 1998). The role of CD59/Neurotoxoin/Ly-6-like protein in the formation of anaemia needs to be determined.

\section{Ubiquitin-proteasome pathway (UPP)-related genes}

Over the last decade, it has become clear that modification of protein by the covalent attachment of ubiquitin and ubiquitin-related protein is involved in the control of many, if not all, fundamental cellular processes (Coscoy \& Ganem 2000). Some viral proteins, as well as being substrates for the UPP, directly target this system for utilization by the virus for its own purposes. First, some viral proteins seem to specifically target major histocompatibility class I molecules for ubiquitination, resulting in the internalization and subsequent degradation of these molecules in the endosomal/lysosomal system (Coscoy \& Ganem 2000). Second, in some viruses, ubiquitination of viral capsid proteins may be needed for the maturation and the release of the viral progeny (Strack et al. 2000). In some situations, the virus utilizes the UPP to target a number of important negative cell regulatory proteins for degradation, which is presumably important for the virus. In ISKNV-infected mandarin fish, polyubiquitin, ubiquitin-activating enzyme E1 and proteasome activator subunit 2 genes were up-regulated. This suggested that the ISKNV might recruit the host UPP for its purposes.

Additionally, the ISKNV ORF 99L sequence was found in the forward library. ORF 99L encodes a protein with C3HC4-type zinc finger (RING finger) domain (He et al. 2001). Many RING finger proteins possess ubiquitin ligase E3 activity (Liu 2004). During ISKNV infection, ORF 99L was suggested to be expressed and act as ubiquitin ligase E3, but its specific substrate remains to be identified.

\section{Apoptosis-related gene}

Replicating viruses may stimulate suicide of the host cell directly or provoke recognition by cytolytic $\mathrm{T}$ cells and NK cells. These immune effector cells induce apoptosis (Tortorella et al. 2000). Apoptosis is a crucial biological process that eliminates virus-infected cells and is considered as an innate response induced to counteract viral infection (Wrzesien-Kus et al. 2004). In many cases, apoptosis has an adverse effect on virus replication. In these situations, viruses may express or induce proteins that can interfere at multiple points in the orderly execution of apoptosis. In this paper, host inhibitor of apoptosis protein (IAP) expression level was increased in spleen cells after ISKNV infection. Mammalian IAPs block apoptosis either by binding and inhibiting caspases or through caspase-independent mechanisms (Wrzesien-Kus et al. 2004). We suggest that the mandarin fish IAP was induced by ISKNV infection and the up-regulation of IAP expression would be advantageous to ISKNV replication. Further work needs to be done to confirm this.

\section{CCAAT/enhancer binding protein beta (C/EBP $\beta)$ gene}

After ISKNV infection, the C/EBP $\beta$ was upregulated. C/EBP $\beta$ are a family of leucine zipper transcription factors comprising 6 members: $\alpha, \beta, \delta, \varepsilon, \gamma$ and $\xi$, that are critically involved in the regulation of normal cellular differentiation and function in multiple tissues (Lekstrom-Himes \& Xanthopoulos 1998). They act as gene regulatory proteins that are either transcriptional repressors or activators (Darlington et al. 1998) and the binding motifs have been identified in regulatory regions of various genes expressed by cells of myelomonocytic lineages, particularly those encoding inflammatory cytokines (IL-6, TNF- $\alpha$ ), other cytokines (IL-8 and IL-12) and genes encoding proteins important for macrophage and granulocytic function, including macrophage, granulocyte and granulocyte/macrophage receptor genes (Poli 1998), consequently reflecting the biological significance of C/EBPs as regulators of immune related genes.

C/EBPs bind to virus promoters or proteins and play a role in virus replication. Wang et al. (2003a,b) recently demonstrated that $\mathrm{C} / \mathrm{EBP} \alpha$ is able to reciprocally transactivate both replication-associated protein and replication and transcription activator promoters of Kaposi's sarcoma-associated herpesvirus (KSHV), and that the introduction of exogenous $\mathrm{C} / \mathrm{EBPq}$ induces mRNA and protein expression from both of these immediate-early genes in endogenous latent genomes in KSHV-infected PEL cell lines. Furthermore, $\mathrm{C} / \mathrm{EBP} \alpha$ was reported to bind to the Epstein-Barr virus ZTA protein during induction of the EBV lytic cycle (Wu et al. 2004). C/EBP $\beta$ was reported to bind to HIV LTR and repress the transcription of HIV (Nakata 2000). The function of mandarin fish C/EBP will be determined in future research. 


\section{Ribosomal protein genes}

Iridovirus FV3 infection results in a rapid inhibition of host cell protein synthesis. Translational shut-off appears to be consequence of a complex series of events involving the degradation of host mRNA (Chinchar 2002) but the degraded mRNAs during virus infection were not identified in detail. mRNAs encoding ribosomal proteins in HeLa cells undergo concomitant degradation after infection with herpes simplex virus type 1 (Greco et al. 1997). In this paper, ribosomal protein ESTs L17, L10, L22, L19, L30, L21, S17, S3a, S14, S23, S19, S8, S5, S29 and S7 were identified from the reverse library, which meant the lower mRNA level in ISKNV-infected fish than in mock-infected fish. The ISKNV infection may degrade the mRNA of these genes, inhibiting the synthesis of host cell proteins. Further studies should be done to confirm this.

As SSH can yield false positives, up- or down-regulation of individual gene transcription will require further detailed investigation.

In summary, the down- or up-regulated gene expression profile in the spleen of mandarin fish after ISKNV infection shows that multiple processes are involved in the infection. These results provide insights into individual genes and pathways that constitute the host cell's response to virus infection. Some cDNA sequences responding to virus infection will be of great value in understanding the immune system of the fish and interactions between the fish and ISKNV. Further studies of some of these proteins and processes are currently ongoing.

Acknowledgements. This research was supported by the Chinese National '863' Project (grant no. 2003AA603011), National Natural Science Foundation of China (grant no. 30300266, 30325035) and funds from the Science and Technology Bureau of Guangdong Province.

\section{LITERATURE CITED}

Altmann SM, Mellon MT, Distel DL, Kim CH (2003) Molecular and functional analysis of an interferon gene from the zebrafish, Danio rerio. J Virol 77:1992-2002

Aries SP, Schaaf B, Hansen F, Weyrich K, Kurowski V, Dennin R, Dalhoff K (1997) Expression of complement receptors and regulatory proteins on alveolar CD4+ lymphocytes from human immunodeficiency virus-1 infected individuals. Eur Respir J 10:1736-1741

Benson DA, Karsch-Mizrachi I, Lipman DJ, Ostell J, Rapp BA, Wheeler DL (2000) GenBank. Nucleic Acids Res 28:15-18

Chen XH, Lin KB, Wang XW (2003) Outbreaks of an iridovirus disease in maricultured large yellow croaker, Larimichthys crocea (Richardson), in China. J Fish Dis 26: 615-619

Chinchar VG (2002) Ranaviruses (family Iridoviridae): emerging cold-blooded killers. Arch Virol 147:447-470
Chou HY, Hsu CC, Peng TY (1998) Isolation and characterization of a pathogenic iridovirus from cultured Grouper (Epinephelus sp.) in Taiwan. Fish Pathol 33:201-206

Chua HC, Ng ML, Woo JJ, Wee JY (1994) Investigation of outbreaks of a novel disease, 'Sleepy Grouper Disease', affecting the brown-spotted grouper, Epinephelus tauvina Forskal. J Fish Dis 17:417-427

Coscoy L, Ganem D (2000) Kaposi's sarcoma-associated herpesvirus encodes two proteins that block cell surface display of MHC class I chains by enhancing their endocytosis. Proc Natl Acad Sci USA 97:8051-8056

Danayadol Y, Direkbusarakom S, Boonyaratpalin S, Miyazaki T, Miyata M (1996) An outbreak of iridovirus-like infection in brown-spotted grouper (Epinephelus malabaracus) cultured in Thailand. AAHRI (Aquatic Animal Health Research Institute) Newsletter 5:6

Darlington GJ, Ross SE, MacDougald OA (1998) The role of $\mathrm{C} / \mathrm{EBP}$ genes in adipocyte differentiation. J Biol Chem 273:30057-30060

Diatchenko L, Lau YF, Campbell AP, Chenchik A and 7 others (1996) Suppression subtractive hybridization: a method for generating differentially regulated or tissue-specific cDNA probes and libraries. Proc Natl Acad Sci USA 93: 6025-6030

Fisicaro N, Aminian A, Hinchliffe SJ, Morgan BP, Pearse MJ, D'Apice AJ, Cowan PJ (2000) The pig analogue of CD59 protects transgenic mouse hearts from injury by human complement. Transplantation 70:963-968

Greco A, Laurent AM, Madjar JJ (1997) Repression of betaactin synthesis and persistence of ribosomal protein synthesis after infection of HeLa cells by herpes simplex virus type 1 infection are under translational control. Mol Gen Genet 256:320-327

He JG, Weng SP, Huang ZJ, Zeng K (1998) Identification of outbreak and infectious diseases pathogen of Siniperca chuatsi. Acta Scientiarum Naturalium Universitatis Sunyatseni 5:74-77

He JG, Weng SP, Zeng K, Huang ZJ, Chan SM (2000) Systemic disease caused by an iridovirus-like agent in cultured mandarinfish, Siniperca chuatsi (Basilewsky), in China. J Fish Dis 23:219-222

He JG, Deng M, Weng SP, Li Z, Zhou SY, Long QX, Wang XZ, Chan SM (2001) Complete genome analysis of the mandarin fish infectious spleen and kidney necrosis iridovirus. Virology 291:126-139

He JG, Zeng K, Weng SP, Chan SM (2002) Experimental transmission, pathogenicity and physical-chemical properties of infectious spleen and kidney necrosis virus (ISKNV). Aquaculture 204:11-24

Inouye K, Yamano K, Maeno Y, Nakajima K, Matsuoka M, Wada Y, Sorimachi M (1992) Iridovirus infection of cultured red sea bream, Pagrus major. Fish Pathol 27:19-27

Jung SJ, Oh MJ (2000) Iridovirus-like infection associated with high mortalities of striped beakperch, Oplegnathus fasciatus (Temmink et Schlegel), in southern coastal areas of the Korean peninsula. J Fish Dis 23:223-226

Kawano M, Tsunoda S, Koni I, Mabuchi H, Muramoto H, Yachie A, Seki H (1997) Decreased expression of 20-kD homologous restriction factor (HRF20, CD59) on T lymphocytes in Epstein-Barr virus (EBV)-induced infectious mononucleosis. Clin Exp Immunol 108:260-265

Lekstrom-Himes J, Xanthopoulos KG (1998) Biological role of the CCAAT/enhancer-binding protein family of transcription factors. J Biol Chem 273:28545-28548

Leong JC, Trobridge GD, Kim CH, Johnson M, Simon B (1998) Interferon-inducible Mx proteins in fish. Immunol Rev 166:349-363 
Liu YC (2004) Ubiquitin ligases and the immune response. Annu Rev Immunol 22:81-127

Long S, Wilson M, Bengten E, Bryan L, Clem LW, Miller NW, Chinchar VG (2004) Identification of a cDNA encoding channel catfish interferon. Dev Comp Immunol 28:97-111

Lutfalla G, Crollius HR, Stange-Thomann N, Jaillon O, Mogensen K, Monneron D (2003) Comparative genomic analysis reveals independent expansion of a lineage-specific gene family in vertebrates: the class II cytokine receptors and their ligands in mammals and fish. BMC Genomics 4:29. Available at: www.biomedcentral.com/ 1471-2164/4/29

McGrogan DG, Ostland VE, Byrne PJ, Ferguson HW (1998) Systemic disease involving an iridovirus-like agent in cultured tilapia, Oreochromis niloticus L.-a case report. J Fish Dis 21:149-152

Nakajima K, Sorimachi M (1995) Production of monoclonal antibodies against red sea bream iridovirus. Fish Pathol 30:47-52

Nakata K (2000) [Tuberculosis and the human immunodeficiency virus infection]. Nihon Hansenbyo Gakkai Zasshi 69:87-92 (in Japanese)

Poli V (1998) The role of C/EBP isoforms in the control of inflammatory and native immunity functions. J Biol Chem 273:29279-29282

Rautemaa R, Helander T, Meri S (2002) Herpes simplex virus 1 infected neuronal and skin cells differ in their susceptibility to complement attack. Immunology 106:404-411

Robertsen B, Bergan V, Rokenes T, Larsen R, Albuquerque A (2003) Atlantic salmon interferon genes: cloning, sequence analysis, expression, and biological activity. J Interferon Cytokine Res 23:601-612

Rodge HD, Kobs M, Macartney A, Frerichs GN (1997) Systemic iridovirus infection in freshwater angelfish, Pterophyllum scalare (Lichtenstein). J Fish Dis 20:69-72

Shi CY, Wang YG, Yang SL, Huang J, Wang QY (2004) The first report of an iridovirus-like agent infection in fanned turbot, Scophthalmus maximus, in China. Aquaculture 236:11-25

Strack B, Calistri A, Accola MA, Palu G, Gottlinger HG (2000) A role for ubiquitin ligase recruitment in retrovirus release. Proc Natl Acad Sci USA 97:13063-13068

Sudthongkong C, Miyata M, Miyazaki T (2002a) Iridovirus disease in 2 ornamental tropical freshwater fishes: African lampeye and dwarf gourami. Dis Aquat Org 48:163-173

Sudthongkong C, Miyata M, Miyazaki T (2002b) Viral DNA sequences of genes encoding the ATPase and the major

Editorial responsibility: Jo-Ann Leong,

Kaneohe, Hawaii, USA capsid protein of tropical iridovirus isolates which are pathogenic to fishes in Japan, South China Sea and Southeast Asian countries. Arch Virol 147:2089-2109

Tortorella D, Gewurz BE, Furman MH, Schust DJ, Ploegh HL (2000) Viral subversion of the immune system. Annu Rev Immunol 18:861-926

Wang SE, Wu FY, Fujimuro M, Zong J, Hayward SD, Hayward GS (2003a) Role of CCAAT/enhancer-binding protein alpha (C/EBPalpha) in activation of the Kaposi's sarcoma-associated herpesvirus (KSHV) lytic-cycle replication-associated protein (RAP) promoter in cooperation with the KSHV replication and transcription activator (RTA) and RAP. J Virol 77:600-623

Wang SE, Wu FY, Yu Y, Hayward GS (2003b) CCAAT/ enhancer-binding protein-alpha is induced during the early stages of Kaposi's sarcoma-associated herpesvirus (KSHV) lytic cycle reactivation and together with the KSHV replication and transcription activator (RTA) cooperatively stimulates the viral RTA, MTA, and PAN promoters. J Virol 77:9590-9612

Wang YQ, Lü L, Weng SP, Huang JN, Chan SM, He JG (2006) Molecular epidemiology and phylogenetic analysis of a marine fish infectious spleen and kidney necrosis viruslike (ISKNV-like) virus. Arch Virol (in press) doi: 10.1007/s00705-006-0870-4

Weng SP, He JG, Zeng K, Huang ZJ (1998) Infectious spleen and kidney necrosis virus infection in Siniperca chuatsi (Basilersky) - histopathology and relationship with $\mathrm{HB}$, RBC, and WBC. South China Normal University, Natural Science 82:71-89

Weng SP, Wang YQ, He JG, Deng M, Lu L, Guan HJ, Liu YJ, Chan SM (2002) Outbreaks of an iridovirus in red drum, Sciaenops ocellata (L.), cultured in southern China. J Fish Dis 25:681-685

Williams T (1996) The iridoviruses. Adv Virus Res 46:345-412

Wrzesien-Kus A, Smolewski P, Sobczak-Pluta A, Wierzbowska A, Robak T (2004) The inhibitor of apoptosis protein family and its antagonists in acute leukemias. Apoptosis 9:705-715

Wu FY, Wang SE, Chen H, Wang L, Hayward SD, Hayward GS (2004) CCAAT/enhancer binding protein alpha binds to the Epstein-Barr virus (EBV) ZTA protein through oligomeric interactions and contributes to cooperative transcriptional activation of the ZTA promoter through direct binding to the ZII and ZIIIB motifs during induction of the EBV lytic cycle. J Virol 78:4847-4865

Submitted: October 1, 2005; Accepted: July 17, 2006

Proofs received from author(s): November 20, 2006 